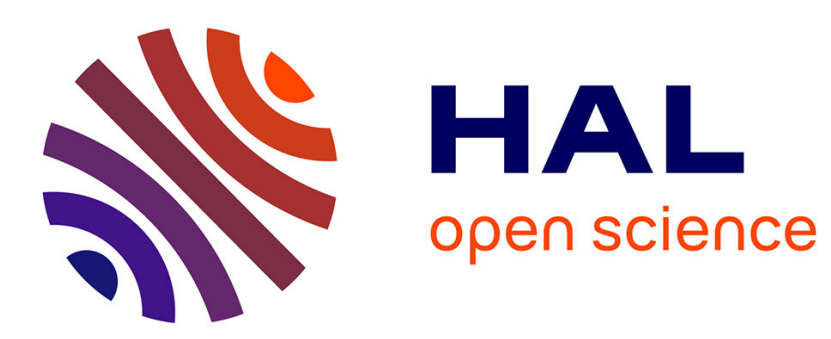

\title{
Boundary singularities of positive solutions of some nonlinear elliptic equations
}

\author{
Marie-Françoise Bidaut-Veron, Augusto C. Ponce, Laurent Veron
}

\section{To cite this version:}

Marie-Françoise Bidaut-Veron, Augusto C. Ponce, Laurent Veron. Boundary singularities of positive solutions of some nonlinear elliptic equations. Comptes rendus de l'Académie des sciences. Série I, Mathématique, 2007, 344, pp.83-88. hal-00281803

\section{HAL Id: hal-00281803 https://hal.science/hal-00281803}

Submitted on 24 May 2008

HAL is a multi-disciplinary open access archive for the deposit and dissemination of scientific research documents, whether they are published or not. The documents may come from teaching and research institutions in France or abroad, or from public or private research centers.
L'archive ouverte pluridisciplinaire HAL, est destinée au dépôt et à la diffusion de documents scientifiques de niveau recherche, publiés ou non, émanant des établissements d'enseignement et de recherche français ou étrangers, des laboratoires publics ou privés. 


\title{
Boundary singularities of positive solutions of some nonlinear elliptic equations
}

\author{
Singularités au bord de solutions positives d'équations elliptiques \\ non-linéaires \\ Marie-Francoise Bidaut-Véron, Augusto C. Ponce, Laurent Véron \\ Laboratoire de Mathématiques et Physique Théorique, CNRS UMR 6083, Faculté des Sciences, 37200 Tours, France
}

\begin{abstract}
We study the behavior near $x_{0}$ of any positive solution of $(\mathrm{E})-\Delta u=u^{q}$ in $\Omega$ which vanishes on $\partial \Omega \backslash\left\{x_{0}\right\}$, where $\Omega \subset \mathbb{R}^{N}$ is a smooth domain, $q \geq(N+1) /(N-1)$ and $x_{0} \in \partial \Omega$. Our results are based upon a priori estimates of solutions of (E) and existence, non-existence and uniqueness results for solutions of some nonlinear elliptic equations on the upper-half unit sphere. To cite this article: M.-F. Bidaut-Véron, A.C. Ponce, L. Véron, C. R. Acad. Sci. Paris, Ser. I XXX (2006).

\section{Résumé}

Nous étudions le comportement quand $x$ tend vers $x_{0}$ de toute solution positive de $(\mathrm{E})-\Delta u=u^{q}$ dans $\Omega$ qui s'annule sur $\partial \Omega \backslash\left\{x_{0}\right\}$, où $\Omega \subset \mathbb{R}^{N}$ est un domaine régulier, $q \geq(N+1) /(N-1)$ et $x_{0} \in \partial \Omega$. Nos résultats sont fondés sur des estimations a priori des solutions de $(\mathrm{E})$, et des résultats d'existence, de non existence et d'unicité de solutions de certaines équations elliptiques non linéaires sur la demi-sphère unité. Pour citer cet article : M.-F. Bidaut-Véron, A.C. Ponce, L. Véron, C. R. Acad. Sci. Paris, Ser. I XXX (2006).
\end{abstract}

Version française abrégée Soit $\Omega$ un ouvert régulier de $\mathbb{R}^{N}, N \geq 2$, tel que $0 \in \partial \Omega$. Étant donné $q>1$, nous considérons une fonction $u \in C^{2}(\Omega) \cap C(\bar{\Omega} \backslash\{0\})$ qui vérifie (3). Nous nous intéressons à la description du comportement de $u$ au voisinage de 0 .

Email addresses: veronmf@univ-tours.fr (Marie-Francoise Bidaut-Véron), ponce@lmpt.univ-tours.fr (Augusto C. Ponce), veronl@univ-tours.fr (Laurent Véron). 
Nous distinguerons les trois valeurs critiques de $q$ données par (4). Si $1<q<q_{1}$, le comportement en 0 des solutions est décrit dans [4]; aussi supposerons-nous le plus souvent $q \geq q_{1}$. Si $u$ est une solution de (3) dans $\mathbb{R}_{+}^{N}$ de la forme $u(x)=u(r, \sigma)=r^{-2 /(q-1)} \omega(\sigma)$, alors $\omega$ vérifie l'équation (6). Dans ce cas, nous avons le résultat suivant :

Théorème 0.1 ( $i)$ Si $1<q \leq q_{1}$, le problème (3) n'admet aucune solution.

(ii) Si $q_{1}<q<q_{3}$, (3) admet une unique solution, notée $\omega_{0}$.

(iii) Si $q \geq q_{3}$, (3) n'admet aucune solution.

Le résultat d'unicité décrit en $(i i)$ est en fait un cas particulier d'un résultat plus général :

Théorème 0.2 Pour tous $q>1$ et $\lambda \in \mathbb{R}$, il existe au plus une solution positive de (7).

Ce résultat demeure si, dans $(7), S_{+}^{N-1}$ est remplacé par une boule dans $\mathbb{R}^{N}$, et $\Delta^{\prime}$ par le laplacien ordinaire.

Par simplicité, nous pouvons supposer que $\partial \mathbb{R}_{+}^{N}$ est l'hyperplan tangent à $\Omega$ en 0 . Le théorème ci-dessous donne une classification des singularités isolées du problème (3) :

Théorème 0.3 Soit $q \geq q_{1}$, avec $q \neq q_{2}$. Supposons que la solution $u$ du problème (3) vérifie

$$
0 \leq u(x) \leq C|x|^{-2 /(q-1)} \quad \forall x \in \Omega \cap B_{a}(0),
$$

pour $C, a>0$. Si $q_{1} \leq q<q_{3}$, ou bien $u$ est continue en 0 , ou bien

$$
u(r, \sigma)= \begin{cases}r^{-(N-1)}(\log (1 / r))^{\frac{1-N}{2}}\left(k_{N} \sigma_{1}+o(1)\right) & \text { si } q=q_{1}, \\ r^{-2 /(q-1)}\left(\omega_{0}(\sigma)+o(1)\right) & \text { si } q_{1}<q<q_{3},\end{cases}
$$

lorsque $r \rightarrow 0$, uniformément par rapport à $\sigma \in S_{+}^{N-1} ; k_{N}$ est une constante qui dépend seulement de $N$. Si $q \geq q_{3}$, u est continue en 0 .

L'estimation a priori (1) est obtenue pour $q_{1} \leq q<q_{2}$ :

Théorème 0.4 Si $q_{1} \leq q<q_{2}$, toute solution u de (3) vérifie (1) pour $C=C(N, q, \Omega)>0$.

Les démonstrations détaillées sont présentées dans [2].

\section{Introduction and main result}

Let $\Omega$ be a smooth open subset of $\mathbb{R}^{N}, N \geq 2$, such that $0 \in \partial \Omega$ and let $q>1$. Assume that $u \in C^{2}(\Omega) \cap C(\bar{\Omega} \backslash\{0\})$ is a solution of

$$
\left\{\begin{aligned}
-\Delta u=u^{q} & \text { in } \Omega, \\
u \geq 0 & \text { in } \Omega, \\
u=0 & \text { on } \partial \Omega \backslash\{0\} .
\end{aligned}\right.
$$

Our goal in this paper is to describe the behavior of $u$ in a neighborhood of 0 .

This problem has similar features with the case where $x_{0} \in \Omega$, which has been studied by GidasSpruck [7]. In our case, we encounter three critical values of $q$ in describing the local behavior of $u$ :

$$
q_{1}:=\frac{N+1}{N-1}, \quad q_{2}:=\frac{N+2}{N-2} \quad \text { if } N \geq 3 \quad \text { and } \quad q_{3}:=\frac{N+1}{N-3} \quad \text { if } N \geq 4 .
$$


When $1<q<q_{1}$, it is proved in [4] that for every solution $u$ of (3) there exists $\alpha \geq 0$ (depending on $N$ and $u$ ) such that

$$
u(x)=\alpha|x|^{-N} \rho(x)(1+o(1)) \quad \text { as } x \rightarrow 0,
$$

where $\rho(x)=\operatorname{dist}(x, \partial \Omega), \forall x \in \Omega$. For this reason, we shall mainly restrict ourselves to $q \geq q_{1}$.

Let us first consider the case where $\Omega=\mathbb{R}_{+}^{N}$ and we look for solutions of (3) of the form $u(x)=$ $u(r, \sigma)=r^{-2 /(q-1)} \omega(\sigma)$, where $r=|x|$ and $\sigma \in S_{+}^{N-1}$. An easy computation shows that $\omega$ must satisfy

$$
\left\{\begin{aligned}
-\Delta^{\prime} \omega & =\ell_{N, q} \omega+\omega^{q} & & \text { in } S_{+}^{N-1}, \\
\omega & \geq 0 & & \text { in } S_{+}^{N-1}, \\
\omega & =0 & & \text { on } \partial S_{+}^{N-1},
\end{aligned}\right.
$$

where $\Delta^{\prime}$ denotes the Laplacian in $S^{N-1}$ and $\ell_{N, q}=\frac{2(N-q(N-2))}{(q-1)^{2}}$. Concerning equation (6), we prove

Theorem 1.1 (i) If $1<q \leq q_{1}$, then (6) admits no positive solution.

(ii) If $q_{1}<q<q_{3}$, then (6) admits a unique positive solution.

(iii) If $q \geq q_{3}$, then (6) admits no positive solution.

One of the main ingredients in the proof of Theorem 1.1 (ii) is the following

Theorem 1.2 If $q>1$ and $\lambda \in \mathbb{R}$, then there exists at most one positive solution of

$$
\left\{\begin{aligned}
-\Delta^{\prime} v & =\lambda v+v^{q} & & \text { in } S_{+}^{N-1}, \\
v & =0 & & \text { on } \partial S_{+}^{N-1} .
\end{aligned}\right.
$$

Remark 1 We emphasize that in Theorem 1.2 we do not assume that $q$ is subcritical. The conclusion above remains valid if, in (7), $S_{+}^{N-1}$ is replaced by $B_{1} \subset \mathbb{R}^{N}$ and $\Delta^{\prime}$ by the usual Laplacian in $\mathbb{R}^{N}$. Theorem 1.2 extends a previous result of Kwong-Li [8].

We now return to the case where $\Omega \subset \mathbb{R}^{N}$ is an arbitrary smooth set such that $0 \in \partial \Omega$. For simplicity, we may assume that $\partial \mathbb{R}_{+}^{N}$ is the tangent hyperplane of $\Omega$ at 0 . Using Theorem 1.2, we provide a classification of isolated singularities of solutions of (3):

Theorem 1.3 Let $q \geq q_{1}, q \neq q_{2}$, and let $u$ be a solution of (3). Assume that $u$ satisfies

$$
0 \leq u(x) \leq C|x|^{-2 /(q-1)} \quad \forall x \in \Omega \cap B_{a}(0),
$$

for some $C, a>0$. If $q_{1} \leq q<q_{3}$, then either $u$ is continuous at 0 or

$$
u(r, \sigma)= \begin{cases}r^{-(N-1)}(\log (1 / r))^{\frac{1-N}{2}}\left(k_{N} \sigma_{1}+o(1)\right) & \text { if } q=q_{1}, \\ r^{-2 /(q-1)}\left(\omega_{0}(\sigma)+o(1)\right) & \text { if } q_{1}<q<q_{3},\end{cases}
$$

as $r \rightarrow 0$, uniformly with respect to $\sigma \in S_{+}^{N-1} ; k_{N}$ denotes a constant depending only on $N$ and $\omega_{0}$ is the unique positive solution of (6).

If $q \geq q_{3}$, then $u$ is continuous at 0 .

Remark 2 We do not know whether Theorem 1.3 is true when $q=q_{2}$. In this case, the equation is conformally invariant and thus other techniques are required. If $\Omega=\mathbb{R}_{+}^{N}$, then it can be proved that any solution of (3) depends only on the variables $r=|x|$ and $\theta=\cos ^{-1}\left(x_{1} /|x|\right)$.

The next result establishes the existence of an a priori estimate for the solutions of (3). According to Theorem 1.4 below, assumption (8) is always fulfilled when $q_{1} \leq q<q_{2}$ :

Theorem 1.4 Let $q_{1} \leq q<q_{2}$ and let $u$ be a solution of (3). Then,

$$
0 \leq u(x) \leq C \rho(x)|x|^{-2 /(q-1)-1} \quad \forall x \in \Omega \cap B_{1}(0),
$$

where $C$ depends on $N, q$ and $\Omega$. 
Remark 3 According to the Doob Theorem [6], any positive superharmonic function $v$ in $\Omega$ satisfies $\int_{\Omega}|\Delta v| \rho<\infty$ and admits a boundary trace, which is a Radon measure on $\partial \Omega$. If $u$ is a solution of (3), then its trace must be of the form $k \delta_{x_{0}}$, for some $k \geq 0$. We may have $k>0$ if $1<q<q_{1}$ (see [1]), but $k$ is necessarily equal to 0 if $q \geq q_{1}$. Indeed, by the maximum principle, $u$ satisfies $u \geq k P_{\Omega}(x, 0)$, where $P_{\Omega}$ denotes the Poisson potential of $\Omega$. Since $u^{q} \in L_{\rho}^{1}(\Omega)$ (by the Doob Theorem), we must have $k=0$ if $q \geq q_{1}$.

Detailed proofs will appear in [2].

\section{Sketch of the proofs}

Proof of Theorem 1.1. Assertion (i) is proved by multiplying (6) by $\phi(\sigma)=\sigma_{1}$. Note that $\phi$ is the first eigenfunction of $-\Delta^{\prime}$ on $S_{+}^{N-1}$, with eigenvalue $\lambda_{1}=N-1$. Integrating the resulting expression over $S_{+}^{N-1}$, and using the fact that $1<q \leq q_{1} \Longrightarrow \ell_{N, q} \geq \lambda_{1}$, we obtain $(i)$.

The existence part in $(i i)$ is obtained by using the Mountain Pass Theorem; the uniqueness is a consequence of Theorem 1.2.

Assertion (iii) can be deduced from the following Pohožaev-type identity:

Proposition 2.1 Assume $N \geq 4$ and $q>1$. Then, any solution of (7) satisfies

$$
\begin{aligned}
\frac{N-3}{q+1}\left(q-q_{3}\right) \int_{S_{+}^{N-1}}\left|\nabla^{\prime} v\right|^{2} \phi d \sigma-\frac{(N-1)(q-1)}{q+1}\left(\lambda+\frac{N-1}{q-1}\right) \int_{S_{+}^{N-1}} v^{2} \phi d \sigma & = \\
& =-\int_{\partial S_{+}^{N-1}}\left|\nabla^{\prime} v\right|^{2} d \tau
\end{aligned}
$$

This identity is obtained by computing the divergence of the vector field $P=\left\langle\nabla^{\prime} \phi, \nabla^{\prime} v\right\rangle \nabla^{\prime} v$, where $\nabla^{\prime}$ is the gradient on $S^{N-1}$, and then using the fact that the first eigenfunction satisfies $D^{2} \phi+\phi g_{0}=0$, where $g_{0}$ is the tensor of the standard metric on $S^{N-1}$. In order to establish $(i i i)$, it suffices to observe that $\ell_{N, q} \leq-\frac{N-1}{q-1} \Longleftrightarrow q \geq q_{3}$.

Proof of Theorem 1.2. We first notice that any positive solution of (7) depends only on the variable $\theta=\cos ^{-1}\left(x_{1} /|x|\right) \in[0, \pi / 2]$; this follows from a straightforward adaptation of the Gidas-Ni-Nirenberg moving plane method to $S_{+}^{N-1}$ (see [9]). Thus, $v$ satisfies

$$
\left\{\begin{array}{l}
v^{\prime \prime}+(N-2) \cot \theta v^{\prime}+\lambda v+v^{q}=0 \quad \text { in }(0, \pi / 2), \\
v^{\prime}(0)=0, \quad v(\pi / 2)=0 .
\end{array}\right.
$$

Let $w(\theta):=\sin ^{\alpha} \theta v(\theta)$, where $\alpha>0$. By choosing $\alpha=2(N-2) /(q+3)$, then $w$ satisfies

$$
\left(w^{\prime}(\pi / 2)\right)^{2}=\int_{0}^{\pi / 2} G^{\prime}(\theta) w^{2}(\theta) d \theta
$$

where $G$ is a function of the form $G(\theta)=\sin ^{\beta^{\prime}} \theta\left(\alpha_{1} \sin ^{2} \theta+\alpha_{2}\right)$; the parameters $\alpha_{1}, \alpha_{2}, \beta^{\prime} \in \mathbb{R}$ can be explicitly computed in terms of $\lambda, N$ and $q$.

Assume, by contradiction, that $v_{1}$ and $v_{2}$ are two distinct solutions of (11). Then,

$$
\int_{0}^{\pi / 2} v_{1} v_{2}\left(v_{2}^{q-1}-v_{1}^{q-1}\right) d \theta=0 .
$$

Therefore, their graphs must intersect at some $\theta_{0} \in(0, \pi / 2)$. We claim that $v_{1}$ and $v_{2}$ intersect at least twice in $(0, \pi / 2)$. If there is only one intersection point, then it can be shown that there exists $\gamma \geq 0$ such 
that the function $\theta \mapsto G^{\prime}(\theta)\left(w_{2}^{2}(\theta)-\gamma w_{1}^{2}(\theta)\right)$ never vanishes in $(0, \pi / 2)$. We then let $L(t):=\left(t^{2}-\gamma\right)^{-1}$, $\forall t \in \mathbb{R} \backslash\{\gamma\}$. By (12) and the Mean Value Theorem, there exists $\theta_{1} \in(0, \pi / 2)$ such that

$$
L\left(\frac{w_{2}^{\prime}(\pi / 2)}{w_{1}^{\prime}(\pi / 2)}\right)=\frac{\int_{0}^{\pi / 2} G^{\prime}(\theta) w_{1}^{2}(\theta) d \theta}{\int_{0}^{\pi / 2} G^{\prime}(\theta)\left[w_{2}^{2}(\theta)-\gamma w_{1}^{2}(\theta)\right] d \theta}=L\left(\frac{w_{2}\left(\theta_{1}\right)}{w_{1}\left(\theta_{1}\right)}\right) .
$$

Since $L$ is injective in $\mathbb{R}_{+}$, this implies

$$
\frac{w_{2}^{\prime}(\pi / 2)}{w_{1}^{\prime}(\pi / 2)}=\frac{w_{2}\left(\theta_{1}\right)}{w_{1}\left(\theta_{1}\right)}
$$

On the other hand, by the Sturm-Liouville Theory, the function $\theta \mapsto w_{2}(\theta) / w_{1}(\theta)$ is (strictly) monotone. L'Hôpital's Rule yields a contradiction as we let $\theta \rightarrow \pi / 2$. Therefore, $v_{1}$ and $v_{2}$ must intersect at least twice. This fact leads to another contradiction by using the Shooting Method (see [8]). Thus, $v_{1}=v_{2}$ in $(0, \pi / 2)$.

Remark 4 The method above follows the lines of the proof of Kwong-Li [8]. The main difference is that we use an alternative argument based on the Mean Value Theorem in order to deduce (14). In [8], they have to assume that the exponent $q$ is subcritical.

Proof of Theorem 1.3. It follows from methods developed in [7] and [3]. For simplicity, we shall assume that $a=1$ and $\partial \Omega \cap B_{1}=\partial \mathbb{R}_{+}^{N} \cap B_{1}$. We set

$$
w(t, \sigma)=r^{2 /(q-1)} u(r, \sigma), \quad t=\log (1 / r) \in(0, \infty) \times S_{+}^{N-1}:=Q .
$$

Then, $w$ satisfies

$$
w_{t t}-\left(N-2 \frac{q+1}{q-1}\right) w_{t}+\Delta^{\prime} w+\ell_{N, q} w+w^{q}=0 \quad \text { in } Q
$$

and $w$ vanishes on $(0, \infty) \times \partial S_{+}^{N-1}$. Since $w$ is uniformly bounded on $Q$, standard a priori estimates for elliptic problems yield

$$
\left|\partial_{t}^{k} \nabla^{\prime j} w\right| \leq M_{k, j} \quad \text { in }(1, \infty) \times S_{+}^{N-1}
$$

for any integers $k, j \geq 0$, where $\nabla^{\prime j}$ stands for the covariant derivative on $S^{N-1}$. Thus, the trajectory $\mathcal{T}_{w}=\{w(t, \cdot): t \geq 1\}$ is relatively compact in $C^{2}\left(\overline{S_{+}^{N-1}}\right)$. Multiplying (15) by $w_{t}$ and integrating over $S_{+}^{N-1}$, we obtain

$$
\frac{d}{d t} H(t)=\left(N-2 \frac{q+1}{q-1}\right) \int_{S_{+}^{N-1}} w_{t}^{2} d \sigma
$$

where

$$
H(t):=\frac{1}{2} \int_{S_{+}^{N-1}}\left(w_{t}^{2}-\left|\nabla^{\prime} v\right|^{2}-\ell_{N, q} w^{2}+\frac{2}{q+1} w^{q+1}\right) d \sigma .
$$

Since $q \neq q_{2}$, we know that $N-2(q+1) /(q-1) \neq 0$. Thus, iterated energy estimates imply that $w_{t}(t, \cdot), w_{t t}(t, \cdot) \rightarrow 0$ in $L^{2}\left(S_{+}^{N-1}\right)$ as $t \rightarrow \infty$. Therefore, the limit set $\Gamma_{w}$ of $\mathcal{T}$ is a connected subset of the set of solutions of (6). By Theorem 1.1, we deduce that

$$
\Gamma_{w}= \begin{cases}\{0\} & \text { if } q=q_{1} \text { or } q \geq q_{3}, \\ \{0\} \text { or }\left\{\omega_{0}\right\} & \text { if } q_{1}<q<q_{3} .\end{cases}
$$

Then, a linearization argument as in [3] leads to the conclusion if $q>q_{1}$.

We now consider the case $q=q_{1}$; we borrow some ideas from [1] and [11]. We first prove, by ODE techniques, that

$$
X(t):=\int_{S_{+}^{N-1}} w(t, \cdot) \phi d \sigma \leq C t^{-(N-1) / 2} .
$$


Using (8) and the boundary Harnack inequality (see [5]), we derive

$$
0 \leq w(t, \sigma) \leq C t^{-(N-1) / 2} \quad \text { in }(1, \infty) \times S_{+}^{N-1} .
$$

Set $\eta(t, \sigma):=t^{(N-1) / 2} w(t, \sigma)$. We verify as above that the limit set $\Gamma_{\eta}$ in $C^{2}\left(\overline{S_{+}^{N-1}}\right)$ of the trajectory $\mathcal{T}_{\eta}$ of $\eta$ is an interval of the form $\left\{\kappa \phi: 0 \leq \kappa_{0} \leq \kappa \leq \kappa_{1}\right\}$. In order to show that $\mathcal{T}_{\eta}$ is reduced to a single point, we prove that $\|r(t, \cdot)\|_{L^{2}} \leq C t^{-1}$, where

$$
r(t, \cdot):=\eta(t, \cdot)-z(t) \phi \quad \text { and } \quad z(t)=\int_{S_{+}^{N-1}} \eta(t, .) \phi d \sigma .
$$

Writing the equation satisfied by $z$ as a non-homogeneous second order linear ODE, we prove that either $z(t) \rightarrow 0$, which implies that $u$ is continuous at 0 , or $z(t) \rightarrow \tilde{k}_{N}$ as $t \rightarrow \infty$, for some constant depending only on $N$.

Proof of Theorem 1.4. It is an application of the Doubling Lemma Method introduced in [10], from which we derive the following local estimate:

Lemma 2.1 Let $1<q<q_{2}$ and let $u$ be a solution of (3). Then, for every $x_{0} \in \partial \Omega \backslash\{0\}$ and $0<R<\left|x_{0}\right|$, we have

$$
0 \leq u(x) \leq C\left(R-\left|x-x_{0}\right|\right)^{-2 /(q-1)} \quad \forall x \in B_{R}\left(x_{0}\right) \cap \Omega,
$$

for some constant $C>0$ depending only on $\Omega$.

Apply this lemma with $x_{0} \in \partial \Omega \backslash\{0\}$ and $R=\left|x_{0}\right| / 2$. Using elliptic regularity theory, we obtain

$$
0 \leq u(x) \leq C \rho(x)|x|^{-2 /(q-1)-1} \quad \forall x \in \Omega \text { such that } 0<\rho(x)<|x| / 2 .
$$

If $\rho(x) \geq|x| / 2$, then we use Gidas-Spruck's internal estimates (see [7]). We thus obtain (10).

\section{References}

[1] M.-F. Bidaut-Véron, Th. Raoux, Asymptotics of solutions of some nonlinear elliptic systems, Comm. Partial Differential Equations 21 (1996), 1035-1086.

[2] M.-F. Bidaut-Véron, A.C. Ponce, L. Véron, in preparation.

[3] M.-F. Bidaut-Véron, L. Véron, Nonlinear elliptic equations on compact Riemannian manifolds and asymptotics of Emden equations, Invent. Math. 106 (1991), 489-539.

[4] M.-F. Bidaut-Véron, L. Vivier, An elliptic semilinear equation with source term involving boundary measures: the subcritical case, Rev. Mat. Iberoamericana 16 (2000), 477-513.

[5] L. Cafarelli, E. Fabes, S. Mortola, S. Salsa, Boundary behavior of nonnegative solutions of elliptic operators in divergence form, Indiana Univ. Math. J. 30 (1981), 621-640.

[6] J. Doob, Classical potential theory and its probabilistic counterpart, Springer, London, 1984.

[7] B. Gidas, J. Spruck, Global and local behavior of positive solutions of nonlinear elliptic equations, Comm. Pure Appl. Math. 34 (1981), 525-598.

[8] M.K. Kwong, Y. Li, Uniqueness of radial solutions of semilinear elliptic equations, Trans. Amer. Math. Soc. 333 (1992), 339-363.

[9] P. Padilla, Symmetry properties of positive solutions of elliptic equations on symmetric domains, Appl. Anal. 64 (1997), 153-169.

[10] P. Poláčik, P. Quittner, Ph. Souplet, Singularity and decay estimates in superlinear problems via Liouville type theorems. Part I: Elliptic equations and systems, Duke Math. J., to appear.

[11] L. Véron, Comportement asymptotique des solutions d'équations elliptiques semi-linéaires dans $\mathbb{R}^{N}$, Ann. Math. Pura Appl. 127 (1981), 25-50. 